\title{
Tongue-Print: A Novel Biometrics Pattern
}

\author{
David Zhang ${ }^{1, *}$, Zhi Liu' ${ }^{2}$, Jing-qi Yan ${ }^{2}$, and Peng-fei Shi ${ }^{2}$ \\ ${ }^{1}$ Biometrics Research Centre, Department of Computing, Hong Kong Polytechnic University, \\ Kowloon, Hong Kong \\ ${ }^{2}$ Institute of Image Processing and Pattern Recognition, Shanghai Jiao Tong University, \\ Shanghai China \\ csdzhang@comp.polyu.edu.hk,\{liu_zhi,jingqiy\}@sjtu.edu.cn
}

\begin{abstract}
The tongue is a unique organ in that it can be stuck out of mouth for inspection, and yet it is otherwise well protected in the mouth and is difficult to forge. The tongue also presents both geometric shape information and physiological texture information which are potentially useful in identity verification applications. Furthermore, the act of physically reaching or thrusting out is a convincing proof for the liveness. Despite these obvious advantages for biometrics, little work has hitherto been done on this topic. In this paper, we introduce this novel biometric and present a verification framework based on the tongue-prints. The preliminary experimental results demonstrate the feasibility of the tongue biometrics.
\end{abstract}

Keywords: Biometrics, tongue-print, verification.

\section{Introduction}

The reliable automatic recognition of identities has long been an attractive goal, with biometrics [1][2], such as fingerprints, palmprints and iris images already being widely used in a number of identity recognition systems. The list of physiological and behavioral characteristics that have so far been developed and implemented in such systems is long and includes the face, iris, fingerprint, palmprint, hand shape, voice, signature and gait. However, how to counter the forge has been the common challenge for the traditional biometrics. Many of the traditional biometrics, however, are unreliable in that features may be forged, for example by using a fake iris. Actually, the tightened security required the noninvasive biometrics that are anticounterfeiting and can provide liveness verification. Accordingly, it is very necessary to find some new biometrics to fill the requirements.

The tongue may offer a solution to this difficulty, having as it does many properties that make it suitable for use in identity recognition. To begin with, the tongue is unique to each person in its shape (see Fig. 1) and in its surface textures (see Fig. 2). Second, the tongue is the only internal organ that can quite normally and easily be exposed for inspection. This is useful because it is the exposed portion of the tongue that carries a great deal of shape and textural information that can be acquired in images that we call "tongue-print". Third, according to our long time observation, the shape of the individual tongue is constant, notwithstanding its instinctive squirm and 
its physiological textures are invariant even as the coating of the tongue changes. Fourth, as the human tongue is contained in the mouth, it is isolated and protected from the external environment, unlike the fingers, for example. Finally, the process of tongue inspection is also a reliable proof of life.

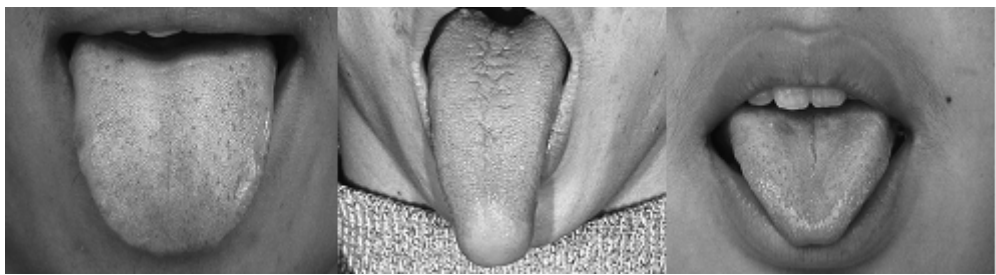

(a)
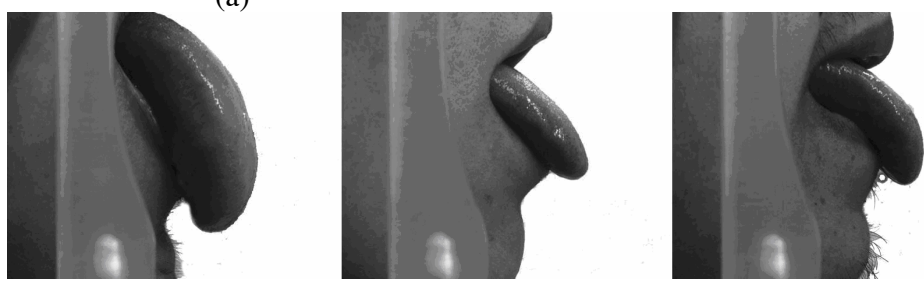

(b)

Fig. 1. Some samples with different shape from frontal and profile view. (a) Different shapes from the frontal view and (b) different shapes from the profile view.

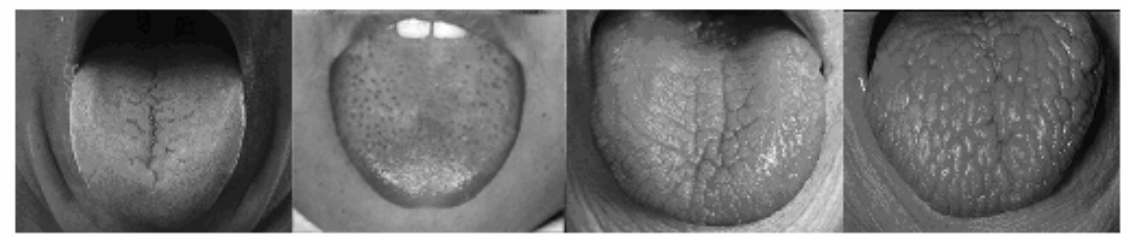

Fig. 2. Some samples with different textures

This promising combination of characteristics was the inspiration for the development of the on-line verification system based on the tongue-prints that is described here. This system extracts both the shape and textural features of the tongue and uses them for recognition. The shape vector represents the shape features of the tongue, specifically its length, bend, thickness, and width, and the curvature of its tip. The texture codes represent the textural features of the central part of the tongue. Fig. 3 gives a block diagram illustrating the framework. The modules are described in the following sections.

The remainder of this paper is organized as follows: Section 2 describes the preprocessing of tongue-print images. Section 3 introduces our feature extraction and recognition framework, describing how we extract shape features and analyze the texture of the tongue. Section 4 presents the experimental results. Section 5 offers our conclusion. 


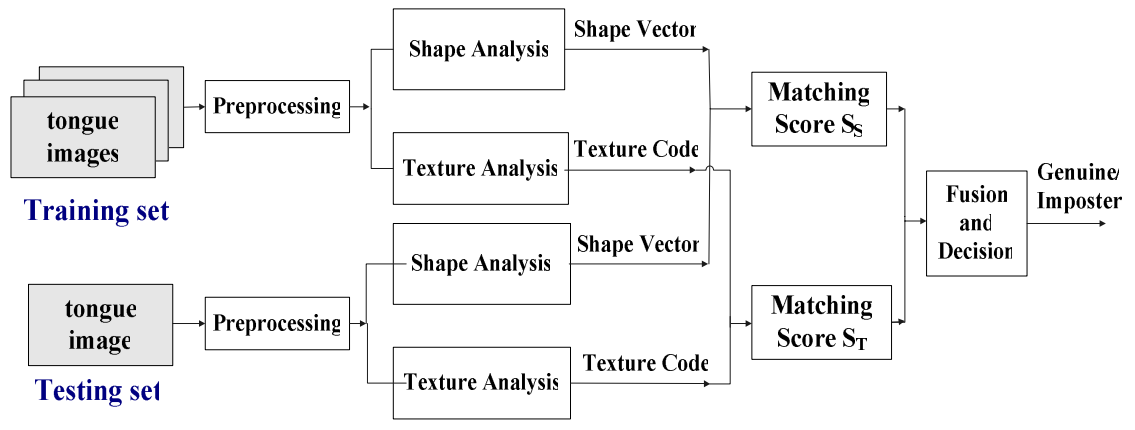

Fig. 3. The block diagram of our tongue-print verification procedure

\section{Tongue Image Preprocessing}

Before feature extraction, it is necessary to preprocess the captured tongue images to obtain an outline of the area of the tongue and to eliminate the non-tongue background. This is done using a tongue contour detection method described in our
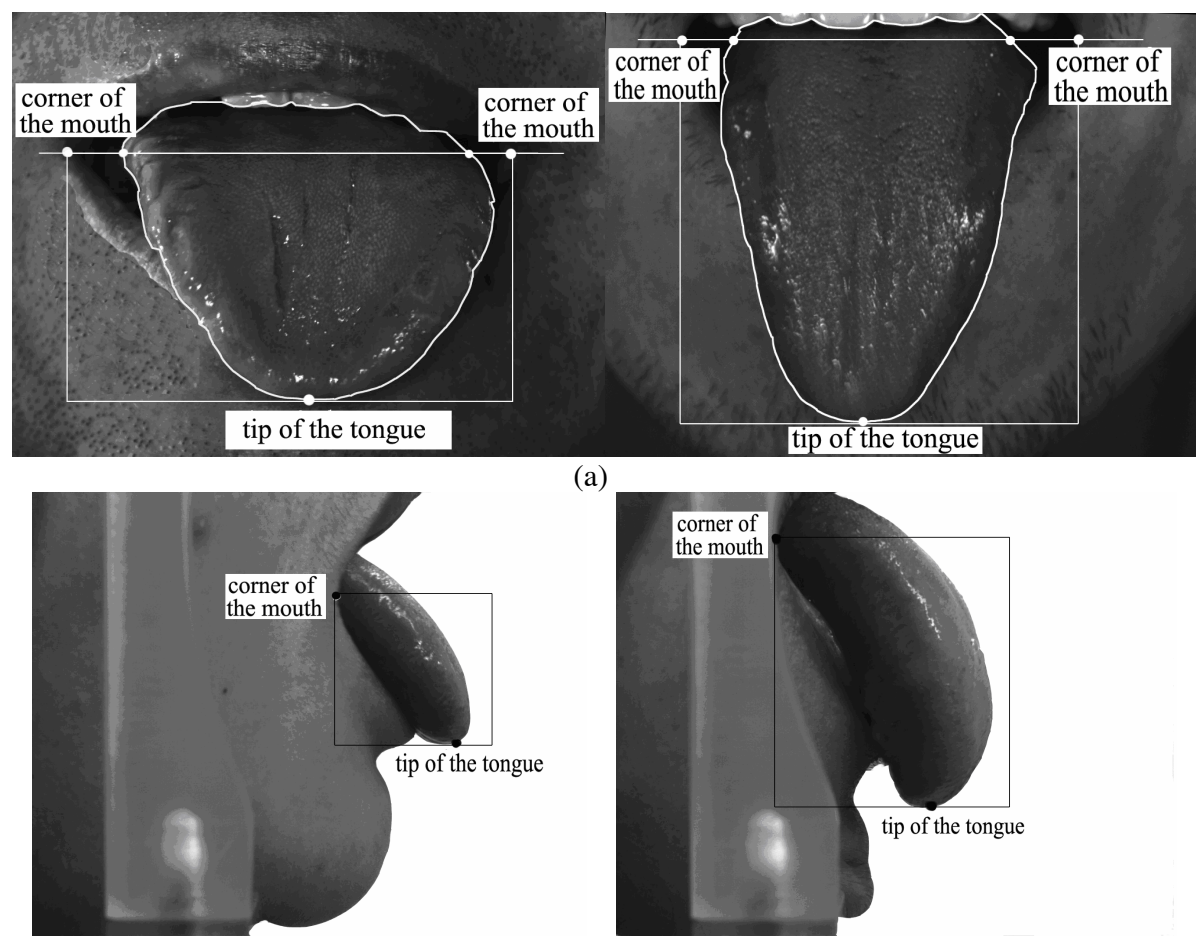

(a)

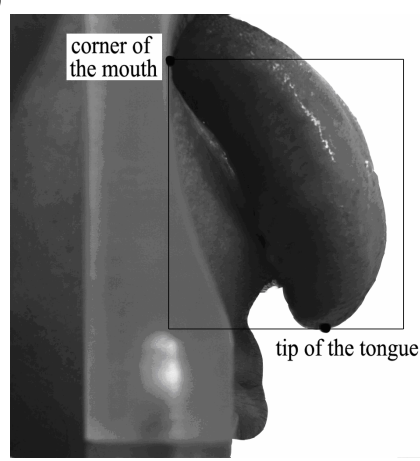

(b)

Fig. 4. The region we determined using corners of the mouth and tip of the tongue. (a) is the frontal view; and (b) is the profile view. 
previous work [7][8] The corners of the mouth and the tip of the tongue (see Fig. 4) can then be used to determine the region of interest (ROI) in the captured tongue image. These ROI provide the main visual features of the tongue that are used in the subsequent procedures.

\section{Tongue-Print Recognition}

In this section we introduce a feature extraction and recognition framework that makes use of both the shape and texture of the tongue. The shape vector represents the geometrical features of the tongue while the texture codes represent the textural features of the central part of the tongue.

\subsection{Shape Feature Extraction}

The shape of the tongue (to be represented as a shape vector) is measured using a set of control points. The control points are $\mathrm{P} 1, \mathrm{P} 2, \ldots, \mathrm{P} 11, \mathrm{P}_{\text {tip }}$ and $\mathrm{P}_{\mathrm{m}}$ (shown in Fig. 5). These control points demarcate important areas of the ROI (here, the part below the segment $\left.L_{P 1, P 2}\right)$. The following describes how five measures, length, bend, thickness, width of the tongue, and the curvature of its tip, are formed as our measurement vectors:

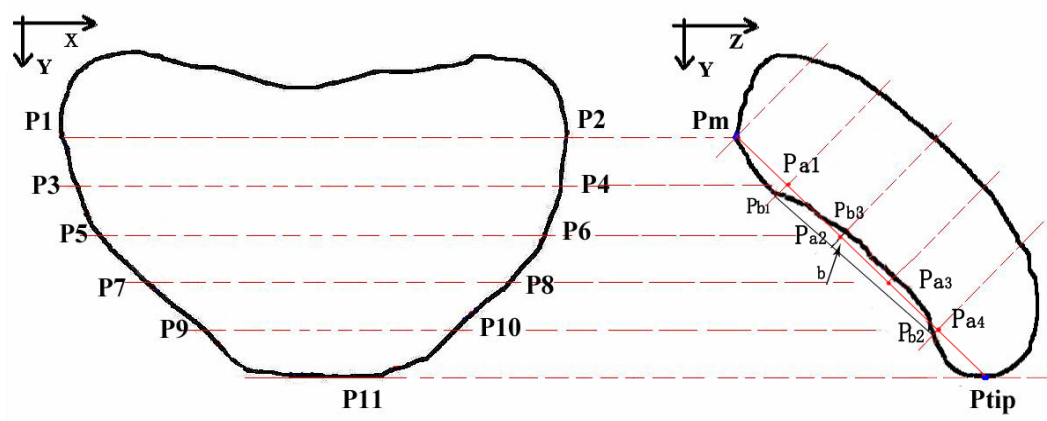

(a)

(b)

Fig. 5. The tongue feature model for the frontal and profile view images. (a) is the frontal view; and (b) is the profile view.

1) Width: We define four segments $\left(L_{P 3, P 4}, L_{P 5, P 6}, L_{P 7, P 8}, L_{P 9, P 10}\right)$ that are parallel to the segment $L_{P 1, P 2}$ in the regions of interest mentioned above. And these segments follow the rule formularized by Eq. (1):

$d\left(L_{P 1, P 2}, L_{P 3, P 4}\right)=d\left(L_{P 3, P 4}, L_{P 5, P 6}\right)=d\left(L_{P 5, P 6}, L_{P 7, P 8}\right)=d\left(L_{P 7, P 8}, L_{P 9, P 10}\right)$

where $d(\bullet)$ represents the distance between two parallel segments. We then use the length of these five segments to construct the width vector $\bar{W}$. 
2) Length: The length of the tongue in the profile view is defined by the distance between $\mathrm{P}_{\text {tip }}$ and $\mathrm{P}_{\mathrm{m}}\left(\mathrm{P}_{\text {tip }}\right.$ denotes the tip of the tongue, and $\mathrm{P}_{\mathrm{m}}$ denotes the corner of the mouth, as shown in Fig. 5 (b)) as follows:

$$
\text { Length }=\left\|P_{\text {tip }}-P_{m}\right\| \text {. }
$$

3) Thickness: Take a line between $P_{\text {tip }}$ and $P_{m}$ (shown in Fig. 5 (b)) and extend the lines ( $L_{P 3, P 4}, L_{P 5, P 6}, L_{P 7, P 8}, L_{P 9, P 10}$ ) so that they intersect with the segment $L_{P_{m} P_{t i p}}$. The points of intersection are labeled as: $P_{a 1}, P_{a 2}, P_{a 3}, P_{a 4}$. Crossing these points, we can get a set of orthogonal lines of the segment $L_{P_{m} P_{t i p}}$. The lengths of these lines within the contour of the profile view are used for the thickness vector $\bar{T}$. In addition, the orthogonal lines that cross $P_{a 1} P_{a 4}$ and $P_{a 2}$ respectively intersect the contour of the tongue at $P_{b 1}, P_{b 2}$ and $P_{b 3}$.

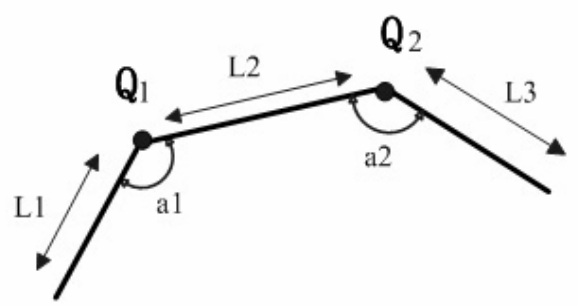

Fig. 6. Total Curvature Measures. L1: length of the segment between $Q_{1}$ and its preceding point; L2: length of the segment between $Q_{1}$ and $Q_{2}$; L3: length of the segment between $Q_{2}$ and its succeeding point; a1: interior angle at $Q_{1}$; a2: interior angle at $Q_{2}$.

4) Curvature of the tip of the tongue: We measure the curvature of the tip of the tongue by using the Total Curvature Function (TCF) [6]. The Total Curvature Function is an approximate estimation method and it is defined for one segment between the two points $Q_{1}$ and $Q_{2}$, as illustrated in Fig. 6. In this figure, the curvature at $Q_{1}$ can be formulated as:

$$
C 1=a 1 /(L 1+L 2)
$$

and the curvature at $Q_{2}$ is formulated as:

$$
C 2=a 2 /(L 2+L 3) .
$$

Thus, the total curvature value of the segment L2 between $Q_{1}$ and $Q_{2}$ is formulated as: 


$$
T C=L 2 *(C 1-C 2) .
$$

We then use these $T C$ to build the vector $\overline{C u r}$ by using the curvature values at the control points $P 3, P 4, \ldots P 9, P 10$ (shown in Fig. 5(a)).

5) Bend: The distance between the middle point $P_{b 3}$ and the segment $L_{P_{b 1} P_{b 2}}$ is formulated as in Eq. (6)

$$
b=D\left(P_{b 3}, L_{P_{b 1} P_{b 2}}\right)
$$

where $D(\bullet)$ computes the distance between the $P_{b 3}$ and $L_{P_{b 1} P_{b 2}}$. Then, we can use $b$ to describe the degree of bend of the tongue. The measurement of $b$ is illustrated in Fig. 5(b).

As the components of these vectors are of different sizes and they have a large dynamic range, it is necessary to normalize them into a single, common range. The five measurement vectors are then combined to form the shape vector that represents the tongue shape.

\subsection{Texture Feature Extraction}

The textural features of the tongue are primarily found on the central part of its surface. To extract this information, we set up a sub-image of the segmented tongue image as a region of interest (ROI). This region is selected under the coordinates system $P_{\text {corner }} O P_{\text {tip }}$ with $256 * 256$ pixels, corresponding to the rectangular area enclosed by the white line in Fig. 7 (a). To extract the texture features, we apply a powerful texture analysis tool, a two dimensional Gabor filter. Gabor filters have been widely used to extract local image features [9][10]. A 2-D Gabor filter in the spatial domain has the following general form [9]:

$$
G(x, y, \theta, u, \sigma)=\frac{1}{2 \pi \sigma^{2}} \exp \left\{\frac{x^{2}+y^{2}}{2 \sigma^{2}}\right\} \exp \{2 \pi \mathrm{i}(u x \cos \theta+u y \sin \theta)\}
$$

where $i=\sqrt{-1} ; u$ is the frequency of the sinusoidal wave; $\theta$ controls the orientation of the function; and $\sigma$ is the standard deviation of the Gaussian envelope. Gabor filters are robust against variations in image brightness and contrast and can be said to model the receptive fields of a simple cell in the primary visual cortex. In order to make the Gabor filter more robust against brightness, it is set to zero DC (direct current) with the application of the following formula [9]:

$$
G^{\prime}(x, y, \theta, \mu, \sigma)=G(x, y, \theta, \mu, \sigma)-\frac{\sum_{i=-n}^{n} \sum_{j=-n}^{n} G(i, j, \theta, \mu, \sigma)}{(2 n+1)^{2}}
$$

where $(2 n+1)^{2}$ is the size of the filter. 


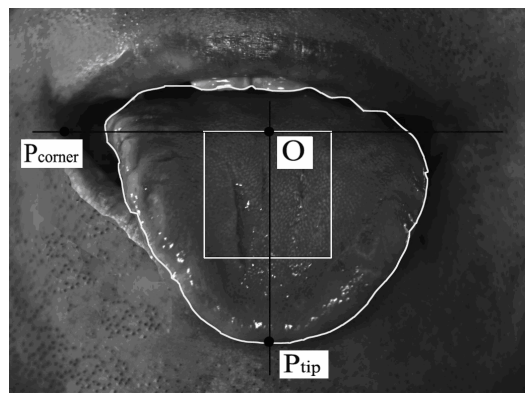

(a)

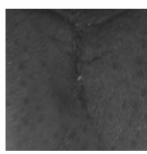

(b)
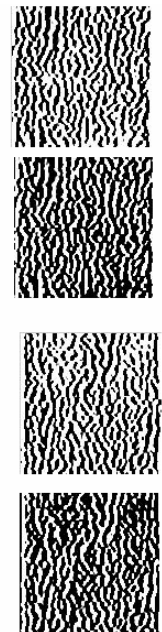

$0^{\circ}$
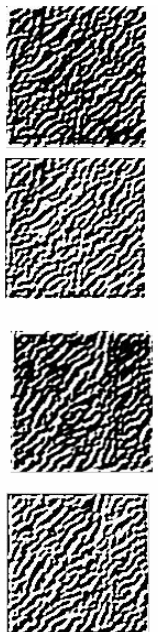

$45^{\circ}$

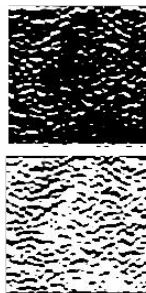

(d)

(e)

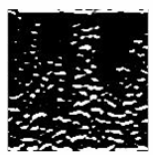

(f)

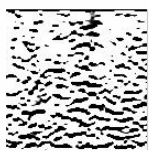

90

Fig. 7. (a) shows the ROI; (b) and (c) are original samples of the textures in the ROI. (d) and (f) are respectively the real parts of features from (b) and(c). (e) and (g) are the imaginary parts of (b) and (c).

An input tongue sub-image $I(x, y), x, y \in \Omega$ ( $\Omega$ is the set of image points) is convolved with $G^{\prime}$. Then, the sample point in the filtered image is coded to two bits, $\left(b_{r}, b_{i}\right)$ using the following rules:

$$
\begin{array}{ll}
b_{r}=1 & \text { if } \operatorname{Re}\left[I \otimes G^{\prime}\right] \geq 0 \\
b_{r}=0 & \text { if } \operatorname{Re}\left[I \otimes G^{\prime}\right]<0 \\
b_{i}=1 & \text { if } \operatorname{Im}\left[I \otimes G^{\prime}\right] \geq 0 \\
b_{i}=0 & \text { if } \operatorname{Im}\left[I \otimes G^{\prime}\right]<0
\end{array}
$$

Using this coding method means that only the phase information in the sub-images is stored in the texture feature vector. This texture feature extraction method was introduced by Daugman for use in iris recognition [11]. Fig. 7 (d)(e)(f)(g) show the features generated in this procedure 


\subsection{Recognition}

In this step, the Mahalanobis distance is used for the tongue shape matching and the Hamming distance is used for the tongue texture code matching. Using these two kinds of distances gives us two matching scores. Because the shape feature vector and texture codes are non-homogeneous and are suitable for different matchers, in this tongue-print based verification method we exploit the matching score level fusion [3]. In our experience, the tongue shape information is more important than texture information. Thus, we apply the following strategy to get the decision results.

$$
S=w_{1} S_{S}+w_{2} S_{T}
$$

where $S$ is the final matching score and the $S_{S}, S_{T}$ are respectively the matching scores in the shape matching module and texture matching module and $w_{1}, w_{2}$ are their corresponding weight values (in our study, $w_{1}=0.6$ and $w_{2}=0.4$ ).

\section{Experiments and Results}

\subsection{Database}

Our database contains 134 subjects. The subjects were recorded in five separate sessions uniformly distributed over a period of five months. Within each session ten image pairs for each subject, a front view and a profile view, were taken using our self-designed tongue-print capture device. In total, each subject provided 50 image pairs. We collected the tongue images from both men and women and across a wide range of ages. The distribution of the subjects is listed in Table 1. We called this tongue image database TB06.

Table 1. Composition of the tongue image database

\begin{tabular}{cccccc}
\hline & \multicolumn{2}{c}{ Sex } & \multicolumn{3}{c}{ Age } \\
\cline { 2 - 6 } & male & female & $20-29$ & $30-39$ & $40-49$ \\
\hline Number of samples & 89 & 45 & 81 & 32 & 21 \\
Percentage (\%) & 66.4 & 33.6 & 60.4 & 23.9 & 15.7 \\
\hline
\end{tabular}

\subsection{Experimental Results}

A matching is counted as a correct matching if two tongue images were collected from the same tongue; otherwise it is an incorrect matching. In our study, the Minimum Distance Classifier [12] is applied for its simplicity. The verification result is obtained using the matching score level fusion [3]. The performance of the verification system when using TB06 is represented by Receiver Operating Characteristic (ROC) curves, which are a plot of the genuine acceptance rate against the false acceptance rate for all possible operating points. From Fig. 8, we can see that combining shape and texture features for the tongue verification produces a better 


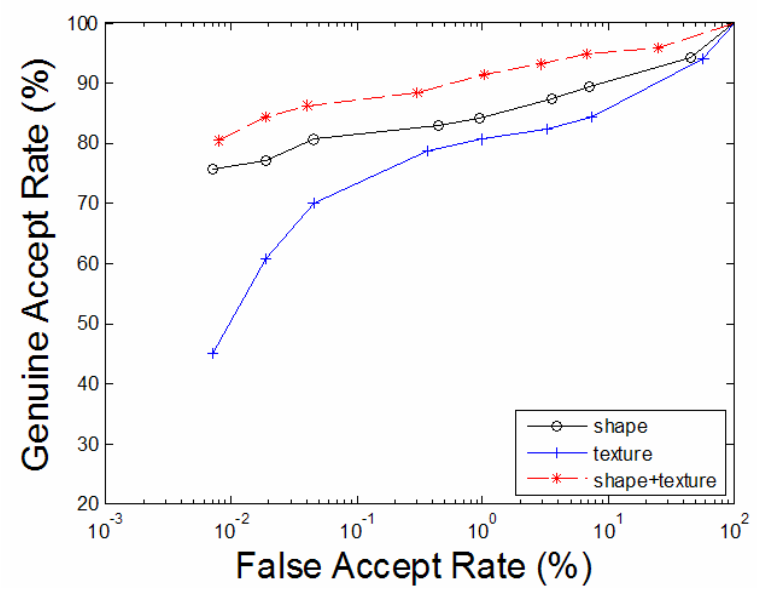

Fig. 8. ROC curve used to illustrate the verification test results

performance than using them singly. When the FAR is equal to $2.9 \%$, we get the Genuine Accept Rate of $93.3 \%$. These results demonstrate that the tongue biometric is feasible.

\section{Conclusions}

As the only internal organ that can be protruded from the body, the human tongue is well protected and is immune to forgery. The explicit features of the tongue cannot be reverse engineered, meaning that tongue verification protects the privacy of users better than other biometrics. This paper presents a novel tongue-print based verification approach. Using a uniform tongue image database containing sample images collected from 134 people, experiments produced a 93.3\% recognition rate. These promising results suggest that tongue-prints of the human tongue qualify as a feasible new member of the biometrics family.

\section{Acknowledgment}

This work was supported in part by the UGC/CRC fund from the HKSAR Government, the central fund from the Hong Kong Polytechnic University and the NSFC fund under the contract No. 60332010.

\section{References}

1. Zhang, D.: Automated Biometrics-Technologies and Systems. Kluwer Academic, Boston (2000)

2. Jain, A., Bolle, R., Pankanti, S.: Biometrics: Personal Identification in Networked Society. Kluwer Academic, Boston (1998) 
3. Ross, A., Jain, A., Qian, J.-Z.: Information Fusion in Biometrics. Pattern Recognition Letters 24(13), 2115-2125 (2003)

4. Hughe, G.E.: On the mean accuracy of statistical pattern recognizers. IEEE Transactions on Information Theory 14(1), 55-63 (1968)

5. Deng, J.-W., Tsui, H.T: A novel two-layer PCA/MDA scheme for hand posture recognition. In: Proceedings of 16th International Conference on Pattern Recognition, vol. 1, pp. 283-286 (2002)

6. Pikaz, A., Dinstein, I.: Matching of partially occluded planar curves. Pattern Recognition 28(2), 199-209 (1995)

7. Pang, B., Zhang, D., Wang, K.Q.: The bi-elliptical deformable contour and its application to automated tongue segmentation in Chinese medicine. IEEE Transactions on Medical Imaging 24(8), 946-956 (2005)

8. zhi, L., Yan, J.-q., Zhou, T., Tang, Q.-1.: Tongue Shape Detection Based on B-Spline. In: ICMLC2006, vol. 6, pp. 3829-3832 (2006)

9. Kong, W.K., Zhang, D., Li, W.: Palmprint feature extraction using 2-D Gabor filters. Pattern Recognition 36(10), 2339-2347 (2003)

10. Jain, A., Healey, G.: A multiscale representation including opponent color features for texture recognition. IEEE Transactions on Image Processing 7(1), 124-128 (1998)

11. Daugman, J.: High confidence visual recognition of persons by a test of statistical independence. IEEE Transactions on Pattern Analysis and Machine Intelligence 15(11), 1148-1161 (1993)

12. Jain, A.K., Duin, R.P.W., Mao, J.: Statistical Pattern Recognition: A Review. IEEE Transactions on Pattern Analysis and Machine Intelligence 22(1), 4-37 (2000) 